\title{
电子商务的信息敏感度分析
}

\section{一 中、美、法三国间的比较研究*}

\section{上海交通大学安泰经济与管理学院 黄 沛 徐海兵**}

黄 沛, 上海交通大学工商管理系主任、教授、博士生导师。毕业于武汉大学商学院, 获经济 学博士学位。1993-96 年在美国 University of Oregon 2002 年 1 月-7 月在加拿大 University of British Columbian，2003 年 7-8 月在加拿大 McGill University 做高级访问学者。曾讲学于香港理工大学， 澳大利亚墨尔本理工大学，新加坡南洋理工大学，清华大学，武汉大学，华中科技大学，东北财 经大学。中国市场学会常务理事，中国高校市场营销研究会常务理事，中国建设银行湖北分行、 河北分行, 交通银行武汉分行, 武汉电信等高级顾问。主要研究方向：市场营销管理模型、市场 竞争战略、市场发展战略理论。曾先后主持项目: 营销渠道变革与模式选择研究 (国家自然科学 基金)、网络空间市场成功与失败因子的定量研究 (国家自然科学基金)、市场波动的混沌动力学 性态研究 (国家社会科学基金) 等 8 项课题 ; 主要著作 : 《国际市场营销》(教育部普通高等教育 "九五"国家级重点教材)、《市场营销学》, 《销售管理》等; 主要论文 : 《模糊偏序关系及其在消费 者广告偏好方面的运用》、《混沌状态下的市场营销问题》、《数据挖掘在市场营销中的应用》等 30 多篇核心期刊论文。获省、部级二等奖 4 项，交大 MBA 优秀教学成果特等奖，交大优秀教师 奖 , 上海市“精品课程”奖。

摘 要：我们称消费者对个人资料的敏感程度为“信息敏感度”。本文通过对中、美、法三国消费者 的调查研究, 阐明这三国消费者信息敏感度的差异和相似点, 并考察信息敏感度与电子商务之间的关系。

关键词：信息敏感度 电子商务 比较研究

国际市场营销者为推行有效的全球市场战略，必须了解各个国家消费者消费行为的差异性和相似点。 在当今“信息爆炸”的社会里，人们普遍将互联网作为一种国际市场营销的媒介，因此探讨各国消费者在 网上的交易行为特征具有重要的现实意义。

互联网上的信息一般包括两种类型：个人资料 (私人资料) 和其他资料 (公司或他人资料)。个人资 料的传播会涉及个人隐私和个人资料的安全问题，但其他资料的传播和利用在某种意义上还有利于消费者 的社会生存。为此，本文专门对个人资料的敏感度或称“信息敏感度” 进行深入分析。根据现代金融研究 中心 (中国上海) 的调查资料, 我们选择了中、美、法三个具有代表性的国家作为调查样本, 这是基于以 下考虑：(1) 这三个国家分别位于亚洲、北美、西欧三个不同地区；(2) 这三个国家都代表着鲜明的不同 文化背景，分别隶属于东方文化、盎格鲁撒克逊文化、拉丁文化；(3) 这三个国家各自都有自己本国的母 语。

\footnotetext{
*本文受国家自然科学基金项目 (项目编号 : 70072011) 资助, 并委托现代金融研究中心 (中国上海) 进行数据的收集和整理。 **徐海兵, 男, 上海交通大学安泰经济与管理学院在读硕士研究生; 主要研究方向 : 市场营销; 通讯地址 : 上海交通大学安 泰经济与管理学院, 邮编 : 200052。
} 


\section{一、个人资料的信息敏感度}

首先本文将个人资料的信息敏感度分成两种类型：(1) 对个人资料本身的敏感度；(2) 对个人资料传 播的敏感度。

（一）对个人资料本身的敏感度

个人资料就其本意而言应属于个人秘密，所以人们对它的使用一般都非常敏感。但进一步分析可以发 现个人资料所包括的种类繁多，消费者对它们的敏感程度也大相径庭。例如，Horible 所作的民意测验表明， “年收入”、“资产”、“所付税额” 等条目是“人们不愿向外界透露的个人资料”，敏感度较高，其次就属 于“家庭和亲属”、“宗教”和“医疗史”等个人资料 (Horible 1999)。

通常来说，“年收入”涉及到个人的地位和财产，是个人比较敏感的资料。但我们发现提交资料所属 的机构不同其敏感度也有所不同。例如，人们不愿意在子亥子所属学校的表格上写明“年收入”，但愿意在 抵押贷款申请表上填写“年收入”。由此可见对于同一资料的敏感度消费者会随着提交资料的机构不同而 不同，这主要是因为每个机构的可靠程度有一定差异。

进一步从三个国家的文化背景来看，一般认为中国消费者的隐私保护意识相对较低 (Horibe 1999)， 所以消费者对个人资料本身的信息敏感度与西方国家相比也相对较低。有人说这是与中国集体主义的文化 氛围相适应 (Hamaguchi 和 Kumon,1992)。在西方“隐私”意识也有差异，美国是最早提出“隐私权”的 国家，而在某些非英语国家里（如法国），词典里甚至没有“隐私”这个单词 (Horibe,1999)，由此可见， 美国消费者对个人资料的敏感度要比法国消费者高。基于上述分析，我们提出以下假设：

假设 1 : 个人财务资料，如收入、资产、债务，是消费者最为敏感的资料。

假设 2 : 对同一项个人资料因所提交的机构不同消费者的敏感度也不同。

假设 3: 美国消费者对个人资料最为敏感，法国消费者次之，中国最后。

(二) 对个人资料传播的敏感度

如今人们在家里就能收到大量的公司邮件或电子信息 , 同时为了购买商品 , 消费者也会向公司提供一 部分必要的个人资料。在这种交易过程中，消费者最关心的是公司如何管理和使用他们的个人资料。事实 上，有些公司会有意无意地透露出一些消费者的个人资料，这就牵涉到消费者对个人资料传播的敏感度问 题。从上面的假设来看，中国消费者对个人隐私权的保护意识要比美国和法国消费者低，因此他们对个人 资料的传播敏感度也会比其他两个国家的消费者低。美国与法国消费者相比，美国已经把隐私权的概念从 原来的被动“独处权”演化为积极的“控制个人资料传播权” (Horibe 1999)，因此就对个人资料传播的敏 感度而言，美国消费者要比法国更强。由此，我们可以提出假设 4 的概念：

假设 4 : 美国消费者对个人资料传播的敏感度最高，法国次之，中国最后。

\section{二、有关信息敏感度的结构分析}

为了从结构上进一步把握个人资料的信息敏感度，我们把对个人资料本身的敏感度和对个人资料传播 的敏感度作为两种属性，将消费者分成四种类型 (见图 1)。

由图 1 可以看出，类型 I 是属于对个人资料本身和对个人资料传播都很敏感的消费者，而类型III则相 反。从结构的划分上我们可以得出如下假设：

假设 5 : 类型 I 的消费者美国最多，法国次之，中国最少，而类型 11 的消费者中国最多，法国次之， 美国最少。 


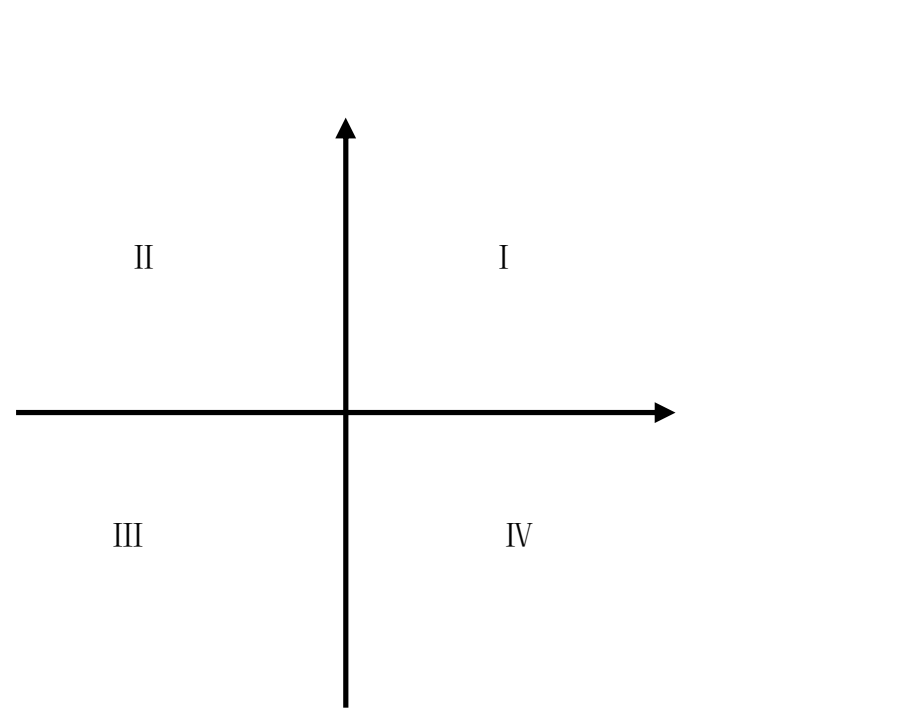

图 1 信息敏感度结构

\section{三、研究方法}

\section{(一) 调查分析}

为了弄清楚中、美、法三国消费者信息敏感度的差异性 ,我们与现代金融研究中心 (中国上海) 于 2000 年 1 月至 2 月, 在中国的上海, 美国的纽约和法国的巴黎 (委托当地的调查机构) 进行了调查。被调查者 男女均有, 年龄范围为 20 至 49 岁。每个国家选取 400 名消费者作为样本, 让调查代理机构利用定额样本 法收集消费者资料。实际收到的样本是, 中国 317, 美国 295, 法国 311。考虑到各国的调查习惯与文化 差异，在中国和美国我们采用信件调查形式，在法国则采用电话调查形式。但两种调查形式所用的问卷是 一样的。问卷主要调查内容包括消费者对个人资料, 隐私, 安全, 互联网, 电子商务等方面的意识和行为。

(二) 对个人资料本身的敏感度

本研究主要考察了 17 个项目 : 性别、生日、地址、电话号码、e-mail 地址、当前住房（已住年限，住 房类型等)、家庭情况 (成员数、组成、政治面貌)、教育背景、工作地 (地址、电话、部门、职称等)、收 入、资产、债务、存款账户号、信用卡数量、宗教、医疗史、业余爱好。关于消费者提交资料的机构我们 设计了 4 种形式：(1) 当地政府或市政团体；(2) 孩子入学的学校；(3) 银行贷款部门；(4) 信用卡公司 或部门。我们询问消费者是否愿意向这 4 种组织机构提交 17 项个人资料。可能的选择有“愿意”、“不愿意” 和“不知道”。为了计算的方便，我们只选择了回答“不愿意” 的信息条目数。各国的平均结果见表 1 。

表 1 三个国家个人资料敏感度的平均项目数

\begin{tabular}{l|l|l|l|l}
\hline & & & & \\
\hline 行政机构 & 7.1 & 5.9 & $\mathbf{7 . 5}$ & $* * * *$ \\
\hline 学校 & 6.6 & 6.2 & $\mathbf{7 . 9}$ & $* * * *$ \\
\hline 金融机构 & 4.9 & 3.8 & $\mathbf{6 . 1}$ & $* * * *$ \\
\hline 信用卡公司 & 6.0 & 5.9 & $\mathbf{7 . 5}$ & $* * * *$ \\
\hline
\end{tabular}

注 : $1 . * * * * \mathrm{p}<0.001 ; 2$. 粗体为三国中的最高值。

由表 1 可以看出，三国间对提交个人资料的敏感度有明显的差异。敏感性最大的是法国，中国次之， 美国最后。这似乎有违于假设 3 , 有关这一部分的分析我们将放在“信息敏感度的 4 种类型”一节做进一 步讨论。 
调查发现，在 4 种组织机构中提交给金融机构的敏感度三国都比较低，特别是提交给银行机构的信息 敏感度更低。究其原因主要是所提供的 17 条个人资料, 大部分是金融机构必需的资料, 如收入、资产和 债务等。换言之，金融机构能比其他组织获得更多的个人资料，这并不是因为“机构的可靠性”，而是因 为“提交资料的必要性” 所至。这一结果与我们的假设 2 是相吻合的。我们继续调查了消费者个人资料对 4 种组织机构的敏感度，表 2 揭示了来自三国信用卡公司的调查结果。

表 2 三国消费者对 17 项个人资料的敏感度 (\%) (针对信用卡公司)

\begin{tabular}{|c|c|c|c|c|}
\hline & 中国 & 美国 & 法国 & F-值的显著性 \\
\hline 性别 & 1.0 & 6.4 & 3.5 & $* * *$ \\
\hline 生日 & 1.6 & 5.4 & 5.1 & $* *$ \\
\hline 地址 & 1.6 & 2.7 & 2.9 & \\
\hline 电话号码 & 4.1 & 7.5 & 8.7 & $*$ \\
\hline e-mail 地址 & 18.3 & 27.1 & 26.4 & $* *$ \\
\hline 当前住房 & 18.3 & 9.5 & 14.5 & $* * *$ \\
\hline 家庭情况 & 22.7 & 20.7 & 30.9 & $* * *$ \\
\hline 教育背景 & 41.3 & 25.4 & 34.1 & $* * *$ \\
\hline 工作地 & 25.2 & 9.8 & 30.2 & $* * *$ \\
\hline 收入 & 35.3 & 10.5 & 28.3 & $* * *$ \\
\hline 资产 & 60.9 & 48.5 & 61.4 & $* * *$ \\
\hline 债务 & 59.0 & 38.6 & 66.6 & $* * *$ \\
\hline 存款账户号 & 49.8 & 68.8 & 70.1 & $* * *$ \\
\hline 信用卡数量 & 65.9 & 65.1 & 66.2 & $* * *$ \\
\hline 宗教 & 52.4 & 60.3 & 77.2 & $* * *$ \\
\hline 医疗史 & 54.9 & 68.5 & 84.2 & $* * *$ \\
\hline 业余爱好 & 30.0 & 48.8 & 50.8 & $* * *$ \\
\hline
\end{tabular}

注 : 1. 表中数据为回答“不” 者所占百分比 ; $2 . * * * \mathrm{p}<0.01, * * \mathrm{p}<0.05, * \mathrm{p}<0.1 ; 3$. 粗体为三国中的最高值, $\mathrm{p}<0.1$ 。

由表 2 可见，许多消费者对财务资料的反应是“不愿意”透露，如“资产”、“债务”、“存款账户号”和 “信用卡数量”等。这一结果与本文假设 1 是相吻合的。然而对于“收入”的反应 “不愿意” 透露者并不 是很多，其占比例为 $10.5 \%$ 35.3\%, 这说明消费者向信用卡公司提供个人收入资料在情理上是可以接受的， 因为这是信用卡公司开展工作所必需的资料。“收入”之所以没有其他财务资料那么敏感，主要是因为“收 入” 是一种“流量”资料。财务上的“流量”资料一般没有财务上的“存量”资料（如“资产”或“债务” 等) 那么敏感。由此可见，在个人资料中财务资料是最为敏感的，其中财务中的“存量” 资料更为敏感。

从三国消费者对全部 17 项个人资料的反应来看 (见表 2 中的粗体字母)，中国消费者对“当前住房”、 “教育背景” 和“收入”的拒答率最大，美国消费者相对较低 (显著性水平为 $\mathrm{p}<0.01$ )。而在法国，拒答 率最大的为“宗教”和“医疗史”等指标（显著性水平为 $\mathrm{p}<0.01$ )。

（三）对个人资料传播的敏感度

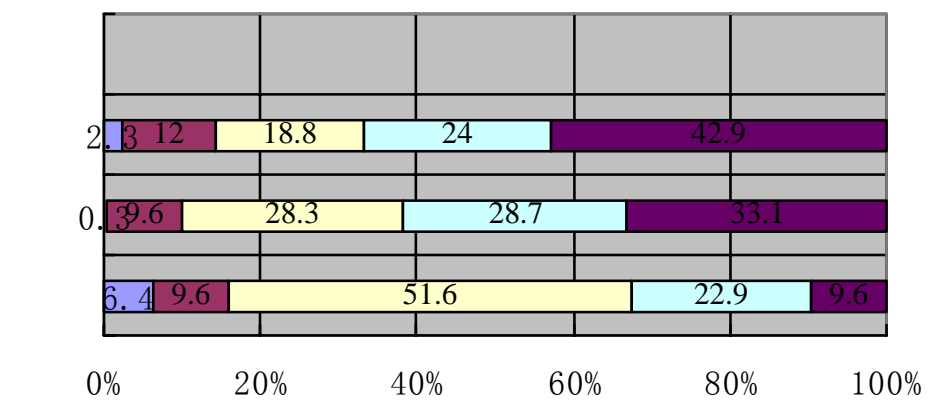

\begin{tabular}{|l|}
\hline$\square$ 很愿意 \\
$\square$ 有些愿意 \\
$\square$ 愿意也不愿意 \\
$\square$ 有些不愿意 \\
$\square$ 不愿意
\end{tabular}

注 : $\chi^{2}$ 检验 $: p<0.01$ 。

图 2 消费者对机构利用其个人资料的反应 
随着一对一营销和关系营销的兴起, 消费者数据库的运用就变得越来越重要了。图 2 表明消费者对公 司建立和使用消费者数据库的反应 $\left(\chi^{2}\right.$ 检验 : $\left.\mathrm{p}<0.01\right)$ 。

如图 2 所示, 在美国和法国, 有 60\%以上 (有些不愿意+不愿意) 的消费者不愿意公司机构利用他们 的个人资料，而且美国所占比例最高 $(66.9 \%)$ 。在中国，这个比例只有 $32.5 \%$ 。这说明我们的假设 4 与实 际结果是相吻合的。

(四) 4 种类型消费者

利用图 1 的结构框架，我们把三个国家的消费者分为 4 种类型。鉴于三个国家所有被访者回答“不愿 意” 的资料平均条目数为 6.29 (在 17 项条目中), 以此我们将横坐标 (对个人资料本身的敏感度) 分开 : 回答资料条目数大于 6.29 的为对个人资料本身高敏感度的消费者, 反之为对个人资料本身低敏感度的消费 者。同时，我们利用与“新”隐私权相关的问题——“让人人都能看到个人资料”和“只能让确保资料可 靠性的机构看到个人资料” 建立纵坐标 (对个人资料传播的敏感度)。如果消费者对两个问题中至少一个 回答“No”，我们就认为他们对个人资料的传播具有高敏感度。如果消费者对两个问题都回答“ Yes”，我 们认为他们对个人资料的传播具有低敏感度。具体结果见以下图 3-5。

(1) 中国

对个人资料传播

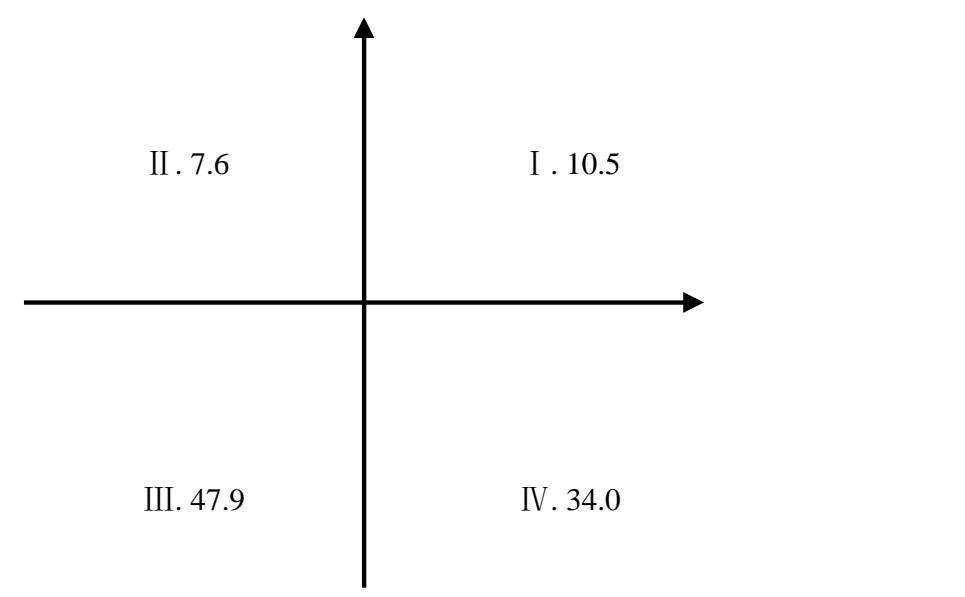

图 3 中国消费者资料敏感度情况

(2) 美国

对个人资料传播

的敏感度

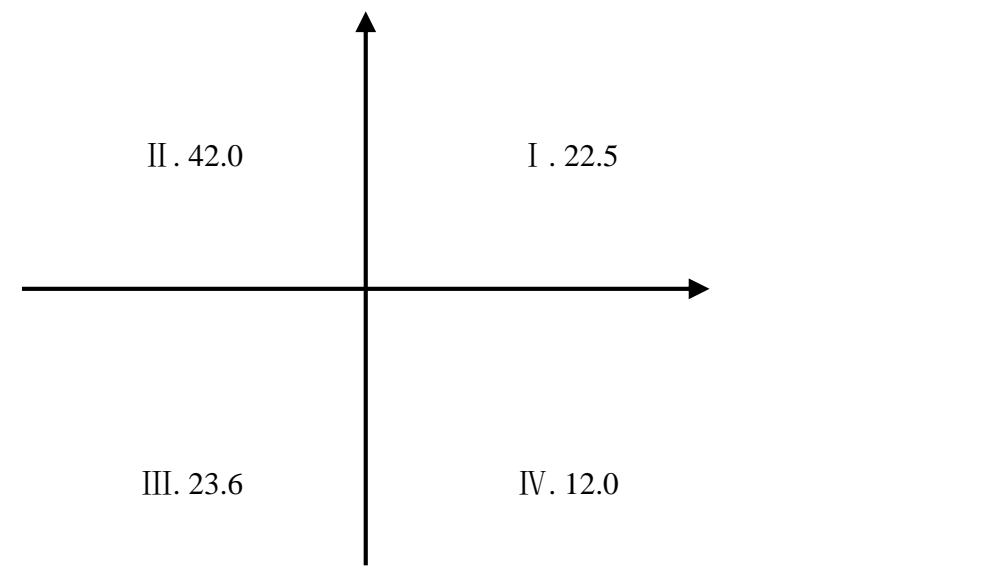

图 4 美国消费者资料敏感度情况 


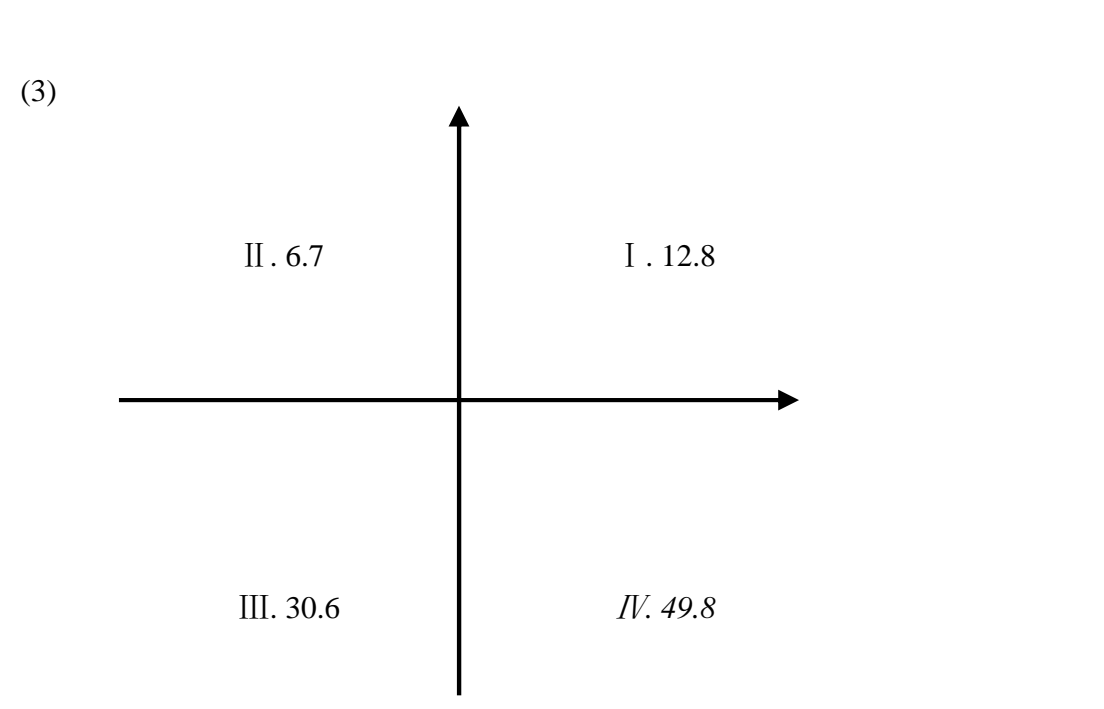

图 5 法国消费者资料敏感度的比较

在图 3-5 中我们用斜体来表示比例最大的消费者类型, 并进行三个国家的比较。首先从第 3 个图示 上我们可以看到近乎 $50 \%$ 的消费者属于类型 III, 这就是说中国消费者几乎有一半不仅对个人资料本身而且 对个人资料传播都不敏感，而且这种不敏感相对于美国和法国而言是相当明显的。这说明中国消费者对信 息敏感度在整体上都比美国和法国消费者低。从第 4 个图示中可以看出美国有 $40 \%$ 以上的消费者属于类型 II，即对个人资料本身不敏感，但对个人资料传播很敏感。这说明美国消费者既有积极的消费意识，也有 维护自身权利的意识。他们同意提交个人资料，但同时又要求对个人资料的传播严格监管。这就是为什么 美国消费者对个人资料敏感度很高的原因，这一点与我们的假设 3 基本吻合。对于法国消费者而言，几乎 $50 \%$ 的消费者属于类型IV, 即对个人资料本身非常敏感, 但对个人资料的传播不很敏感。这说明法国消费 者目前还没进入“新” 隐私权的阶段。

通过消费者结构类型的分析，对假设 5 的进一步解释是，中国消费者普遍的信息敏感度都低，法国消 费者对个人资料本身敏感度较高，而美国消费者对个人资料的传播敏感度较高。如果加进“隐私权”的因 素 : 中国消费者几乎没有隐私权意识, 法国消费者具有传统的、被动的隐私权意识, 而美国消费者表现的 是新的、积极的隐私权意识。

\section{四、信息敏感度与电子商务的关系}

我们调查了三个国家消费者有关互联网或电子商务的意识及其实际使用情况，在此选择了三国中最有 代表性的美国，并列出了信息敏感度和上网意识的分类调查情况（见表 3)。

如表 3 (a) 所示，在 4 种消费者类型中，消费者对网络使用、网上购物、网上支付等方面的反应没有 明显的差异，这说明美国消费者对电子商务活动都持积极的态度。在表 3 (b) 中，4 种类型的消费者对“不 方便、不安全”的反应存在明显的差异。类型VI和 I 的消费者分别占到 $60 \%$ 和 $40 \%$ 之多。这说明越是对个 人资料敏感的消费者, 越是担忧电子商务的安全问题。有关网上服务的问题表 3 (c) 也将 4 种类型的消费 者明显区分开来。其中类型 I 的消费者反应最强烈, 其次是类型 II 。由此可见, 对个人资料传播最敏感的 类型 I 和类型 II 的消费者对电子商务的服务问题最为感兴趣。他们具有一定的“新”隐私权意识, 他们是电 子商务改进服务的“高度相关消费者”。 
电子商务的信息敏感度分析一中、美、法三国间的比较研究

表 3 信息敏感度与网上的行为和意识之间的关系(针对美国消费者)

\begin{tabular}{l|c|c|c|c|c}
\hline & 类型 I & 类型 II & 类型III & 类型IV & F 值水平 \\
\hline (a) 互联网的经验 & & & & & \\
\hline 网络使用 & 54.6 & 52.9 & 43.5 & 54.3 & \\
\hline 网上购物 & 45.0 & 35.9 & 30.0 & 31.6 & \\
\hline 网上支付 & 70.6 & 68.2 & 77.8 & 60.0 & \\
\hline (b) 不上网的原因 & & & & & \\
\hline 不方便、不安全 & 43.3 & 22.2 & 31.6 & $\mathbf{6 0 . 0}$ & $* *$ \\
\hline 没有这个需要 & 40.0 & 50.0 & 36.8 & $\mathbf{8 0 . 0}$ & $* *$ \\
\hline (c) 网上服务的主要使用内容 & & & & & \\
\hline 订票或订旅馆 & $\mathbf{6 4 . 2}$ & 54.5 & 42.1 & 42.3 & 0 \\
\hline 获取金融信息 & $\mathbf{6 2 . 3}$ & 55.5 & 40.4 & 30.8 & $* *$ \\
\hline 建立家庭银行 & $\mathbf{4 5 . 3}$ & 42.6 & 28.1 & 23.1 & 0 \\
\hline 发表意见或工作信息 & $\mathbf{3 4 . 0}$ & 23.8 & 12.3 & 7.7 & $* *$ \\
\hline
\end{tabular}

注 : 1. 数值为回答 “ 是” 的人数所占的百分比 ; $2 . * * \mathrm{p}<0.05, * \mathrm{p}<0.1$;

3. 粗体数值表示三国中的最大值, $\mathrm{p}<0.1 ; 4$. 我们只对 (b) 和 (c) 做了显著性检验。

总而言之，对个人资料本身敏感的消费者对电子商务的接受程度是负相关关系，而对个人资料传播敏 感的消费者对电子商务是正相关关系。至于对个人资料本身低敏感，而对个人资料传播高敏感的类型 II 消 费者，是构成电子商务市场最大的消费者群。

\section{五、结 论}

本文提出了“信息敏感度”概念，这一概念包括两个方面——“对个人资料本身的敏感度”和“对个 人资料传播的敏感度”。根据现代金融研究中心 (中国上海) 所作的关于中、美、法三国消费者的调查资 料，我们找到了这些国家消费者信息敏感度的不同点。中国消费者信息敏感度低; 法国消费者对个人资料 本身敏感; 美国消费者对个人资料传播敏感。我们认为之所以会有这些结果，是因为中国消费者对隐私权 意识比较淡薄; 法国消费者具有的是传统的、被动的隐私权意识; 而美国消费者则开始转向新的、积极的 隐私权意识。

我们只是对信息敏感度和电子商务之间的关系作了初步探索，这主要是受到建立假设资料的限制。值 得注意的是, 我们发现对个人资料本身的敏感度与电子商务的接受存在着负相关关系, 而对个人资料传播 的敏感度与电子商务的接受存在着正相关关系，而对个人资料本身低敏感，对个人资料传播高敏感的类型 II消费者可能构成电子商务市场最大的消费者群。

参考文献 :

1. Culnan, Mary J.. Consumer Awareness of Name Removal Procedures: Implications for Direct Marketing , Journal of Direct Marketing, 1995(9): 10-19

2. Funada, Masayuki. The Protection of Personal Information in the Network Society, The Asia Times (in Chinese), 1998(5): 54-59

3. Horibe, Masao. Privacy and High Level Information Society, Journal of Marketing Research, 1999(6): 35-40

4. Nowak, Glen J. and Joseph Phelps. Understanding Privacy Concerns: An Assessment of Consumers' Information-Related Knowledge and Beliefs, Journal of Direct Marketing, 1997(6): 28-39

5. Http: //www. oecd.org//dsti/sti/ it/ secur /prod/ P RI V EN.HTM

(责任编辑：刘成华、杨帆) 